\title{
Prevalence, awareness, treatment and control of hypertension in Malaysia: A national study of 16,440 subjects
}

\begin{abstract}
Study design: A cross-sectional study was conducted in all states of Malaysia to determine the prevalence, awareness, treatment and control of hypertension. A stratified two-stage cluster sampling design with proportional allocation was used. Methods: Trained nurses obtained two blood pressure measurements from each subject. Hypertension was defined as mean systolic blood pressure $4140 \mathrm{mmHg}$, diastolic blood pressure $490 \mathrm{mmHg}$, or a selfreported diagnosis of hypertension and taking antihypertensive medication. All data were analysed using Stata 9.2 software and took the complex survey design into account. A twosided P-value of o0.05 was considered to be statistically significant. Results: The overall prevalence of hypertension for subjects aged X15 years was $27.8 \%$ (95\% confidence interval (CI) 26.9-28.8). The prevalence of hypertension was significantly higher in males $(29.6 \%$, 95\% CI 28.3-31.0) compared with females (26.0\%, 95\% CI 25.0-27.1). Multivariate logistic regression showed that the odds of having hypertension increased with increasing age, in males, in subjects with a family history of hypertension, with increasing body mass index, in non-smokers and with decreasing levels of education. Only $34.6 \%$ of the subjects with hypertension were aware of their hypertensive status, and 32.4 were taking antihypertensive medication. Amongst the latter group, only $26.8 \%$ had their blood pressure under control. The prevalence of hypertension amongst those aged X30 years has increased from $32.9 \%$ in 1996 to $40.5 \%$ in 2004. Conclusion: In Malaysia, the prevalence of hypertension is high, but levels of awareness, treatment and control are low. There is an urgent need for a comprehensive integrated population-based intervention programme to ameliorate the growing problem of hypertension in Malaysians.
\end{abstract}

Keyword: Hypertension; Epidemiology; Prevalence; Awareness; Treatment; Control; Malaysia. 